\title{
QUALIDADE MICROBIOLÓGICA DO QUEIJO DE COALHO COMERCIALIZADO NO MUNICÍPIO DO CABO DE SANTO AGOSTINHO, PERNAMBUCO, BRASIL
}

\author{
K.A. de Oliveira ${ }^{1 *}$, J. Evêncio Neto ${ }^{1}$, J.E. de Paiva ${ }^{2}$, L.E.H. de Melo ${ }^{3}$ \\ ${ }^{1}$ Universidade Federal Rural de Pernambuco, Departamento de Morfologia e Fisiologia Animal, Rua Dom \\ Manoel de Medeiros s/nº, CEP 52171-900, Recife, PE, Brasil. E-mail: evencioneto@pq.cnpq.br
}

\section{RESUMO}

Este trabalho teve o objetivo de avaliar a qualidade microbiológica do queijo de coalho comercializado no Município do Cabo de Santo Agostinho, Estado de Pernambuco, Brasil, verificando e quantificando micro-organismos patogênicos e indicadores das condições higiênico-sanitárias desse alimento. Foram coletadas 42 (quarenta e duas) amostras de queijo de coalho em 39 (trinta e nove) pontos comerciais distintos, entre janeiro e dezembro de 2008. As amostras foram analisadas pelo Laboratório Central de Pernambuco para determinação do padrão microbiológico do produto, tendo como referência a Resolução da Diretoria Colegiada (RDC) n 12 da Agência Nacional de Vigilância Sanitária. Detectaram-se em 95,24\% (40/42) das amostras discordâncias com os padrões estabelecidos pela RDC12. Com relaçãoa contaminação por coliformes termotolerantes e Staphylococcus coagulase positiva, 80,95\% (34/42) e 76,19\% (32/42), respectivamente, encontravam-se fora dos limites aceitáveis e 9,52\% (4/42) foram positivas para Salmonella sp. Com os resultados obtidos no presente estudo conclui-se que o queijo de coalho comercializado no Município do Cabo de Santo Agostinho, Pernambuco, Brasil apresenta-se em desacordo com os padrões microbiológicos vigentes na legislação brasileira, sendo considerado impróprio para o consumo humano.

PALAVRAS-CHAVE: Alimentos, micro-organismos patogênicos, segurança alimentar, legislação sanitária.

\section{ABSTRACT}

MICROBIOLOGIC QUALITY OF CURD CHEESE SOLD IN THE DISTRICT OF CABO DE SANTO AGOSTINHO, PE, BRAZIL. This study aimed to evaluate the microbiologic quality of curd cheese sold in the district of Cabo de Santo Agostinho (PE), Brazil, by verifying and quantifying pathogenic microorganisms in the product as well as indicators of its hygienic and sanitary conditions. Forty-two samples of cheese collected in 39 different commercial areas were analyzed. The samples were analyzed by the Central Laboratory of Pernambuco, following the procedures established by the Resolution of the Chartered Board of Directors RDC N. 12. It was found that $95.24 \%(40 / 42)$ of the samples of curd cheese presented characteristics that did not meet the standards of RDC 12. As for the contamination of the food by thermotolerant coliforms and coagulase-positive Staphylococcus, 80.95\% (34/42) and 76.19\% (32/42), respectively, did not meet the acceptable standards and 9.52\% (4/42) were positive for Salmonella sp. The results in this study suggest that the curd cheese sold in Cabo de Santo Agostinho, Pernambuco, Brazil, does not comply with the current microbiologic standards prescribed by the Brazilian legislation, which makes it improper for human consumption.

KEY WORDS: Food, pathogenic microorganisms, food safety, sanitary legislation.

\section{INTRODUÇÃO}

O queijo de coalho é produzido há mais de 150 anos, em vários estados da região Nordeste, a partir de leite cru e/ou pasteurizado. Embora constitua um produto popular que faz parte da cultura nordestina, não existe padronização no seu processo de elaboração, sendo comum o emprego de leite cru, o que coloca em risco a saúde do consumidor (CAvalcante et al., 2007).

${ }^{2}$ Universidade Federal Rural de Pernambuco, Departamento de Tecnologia Rural, Recife, PE, Brasil.

${ }^{3}$ Universidade Federal Rural de Pernambuco, Departamento de Medicina Veterinária, Recife, PE, Brasil.

*M.V. Msc. Programa de Pós-Graduação em Ciência Veterinária, Universidade Federal Rural de Pernambuco (UFRPE). 
Entende-se por queijo de coalho o queijo que se obtém por coagulação do leite por meio do coalho ou outras enzimas coagulantes apropriadas, complementada ou não pela ação de bactérias lácteas selecionadas e comercializado normalmente com até dez dias de fabricação (BRASIL, 2001b). Seu nome deve-se ao fato de ter sido tradicionalmente elaborado com leite coagulado pela ação do coalho animal (MANGUEIRA et al., 2002). No Estado de Pernambuco, classifica-se como queijo de coalho tipo " $\mathrm{A}$ " aquele produzido com leite pasteurizado e tipo " $\mathrm{B}$ ", o que é produzido com leite cru (Pernambuco, 1999).

Dentre os queijos de fabricação artesanal no Brasil, o queijo de coalho se destaca como um dos principais e o seu consumo já faz parte do hábito alimentar da população, tanto do Nordeste como, mais recentemente, em inúmeras cidades da região Sudeste. $\mathrm{Na}$ região Nordeste, a produção de queijo de coalho artesanal representa uma atividade de importância social, econômica e cultural (SERviço BRASILEIRO DE Apoio às Micro e Pequenas Empresas, 1994).

Apesar de sua importância econômica e grande popularidade na região Nordeste, a fabricação de queijo de coalho nãoconta com tecnologia apropriada para a melhoria de sua qualidade (Lima et al., 1998).

Alimentos obtidos por processos artesanais têm grande possibilidade de se apresentarem contaminados, devido ao uso de matérias-primas de fontes não seguras, utensílios mal higienizados ou contaminados, elaboração em condições impróprias e armazenamento e comercialização em temperaturas inadequadas, fatores que contribuem para aumentar o risco de causarem enfermidades.

O queijo apresenta características nutritivas que o tornam um alimento muito importante para os humanos, sendo também uma ótima fonte de nutrientes para ocrescimento demicro-organismos, destacandose os coliformes totais, Escherichia coli, Staphylococcus aureus, bactérias mesófilas aeróbicas, bolores e leveduras. Estes, quando presentes nos alimentos, além de reduzirem a qualidade do produto, podem causar danos à saúde do consumidor (SALVADOR et al., 2001).

Estudos realizados por BorGEs et al. (2003) constataram que queijos de coalho produzidos no Estado do Ceará, apresentavam altos níveis de contaminação por bactérias patogênicas, como S. aureus, Salmonella sp. e Listeria monocytogenes.

Do ponto de vista de saúde pública, a população deve ter ao seu alcance alimentos de boa qualidade, dentro de padrões pré-estabelecidos, não só em valores nutritivos, como também quanto às condições higiênicas, que propiciem segurança para a saúde do consumidor (CORREIA; RONCADA, 1997).

Objetivou-secomeste trabalho avaliar a qualidade microbiológica do queijo de coalho comercializado no Município de Cabo de Santo Agostinho, Estado de Pernambuco, Brasil, verificando e quantificando micro-organismos patogênicos e indicadores das condições higiênico-sanitárias desse alimento.

\section{MATERIAL E MÉTODOS}

O estudo foi realizado no Município do Cabo de Santo Agostinho, localizado na região metropolitana do Recife, microrregião Suape, limitando-se ao norte com os municípios de Jaboatão dos Guararapes e Moreno, ao sul com Ipojuca e Escada, ao leste com o Oceano Atlântico e ao oeste com o Município de Vitória deSanto Antão. A área municipal ocupa 446,5 $\mathrm{km}^{2}$, dividida em quatro Regionais, erepresenta $0,45 \%$ do Estado de Pernambuco (СРRM, 2005). A população residente total está estimada em 163.139 habitantes (InATITtuo Brasileiro de GeOgrafia e Estatística, 2007).

As coletas do material em estudo foram realizadas no período de janeiro a dezembro de 2008 nas quatro Regionais do Município do Cabo de Santo Agostinho, totalizando 42 amostras de queijo de coalho, coletadas em 39 estabelecimentos comerciais distintos. Do total das amostras, 34 apresentavam registro no Serviço de Inspeção Estadual e eram do tipo B (produzida com leite cru), uma no Serviço de Inspeção Federal, classificada como tipo A (produzida com leite pasteurizado) e sete não possuíam registro (sem rótulo).

Asamostrasforamanalisadas peloLaboratórioCentraldePernambuco(LACEN/PE) para determinaçãodo padrãomicrobiológicodoalimento, identificandoseelas encontravam-se dentro do limite de aceitabilidade para alimentos destinados ao consumo humano, conformeo estabelecidopela ResoluçãodaDiretoria Colegiada RDC 12 da Agência Nacional de Vigilância Sanitária (BRASIL, 2001a). As análises procederam conforme o descrito pelaAssociação AmericanadeSaúdePública(AmERICAN Public Health Association, 2001) para caracterização de micro-organismos de interesse sanitário em alimentos, sendo pesquisados coliformes termotolerantes, Salmonella sp. e Staphylococcus coagulase positiva.

\section{Contagem de coliformes termotolerantes e Escherichia coli}

O método de análise utilizado foi o do Número Mais Provável (NMP). Para a realização desta técnica utilizou-se como cepa controle positiva E. coli ATCC 25.922. Das colônias típicas de E. coli., duas foram isoladas e submetidas às provas bioquímicas de Indol, Vermelho deMetila, teste deVoges-ProskauereCitrato de Simmons (American Public Health Association, 2001).

\section{Contagem de estafilococos coagulase positiva}

O método de contagem de Staphylococcus coagulase positiva foi o "Spread-plate", onde utilizou-se 
como cepa controle positiva, S. aureus ATCC12.600. A partir das colônias típicas (negras, brilhantes, convexas e rodeadas por zonas claras de 2 a $5 \mathrm{~mm}$ de diâmetro) foram realizadas as provas bioquímicas de catalase e coloração de Gram. Também foi realizada prova bioquímica confirmativa de coagulase (American Public Health Association, 2001).

\section{Pesquisa de Salmonella sp.}

Para este procedimento foi utilizado como cepa controle positiva Salmonella ATCC 14.028. As colônias típicas suspeitas foram submetidas a testes bioquímicos de inoculação em meio IAL (Instituto Adolfo Lutz). Colônias puras foram submetidas à prova de sorologia, utilizando soros polivalentes somáticos e anti-Salmonella flagelar (American Public Health Association, 2001).

\section{RESULTADOS E DISCUSSÃO}

Das 42 amostras analisadas, 40 (95,24\%) apresentaram discordância com os padrões microbiológicos estabelecidos pela RDC 12 (BRASIL, 2001a), sendo consideradas inadequadas para o consumo humano (Tabela 1).

Em relação aos coliformes termotolerantes, constatou-se que $80,95 \%$ (34/42) das amostras apresentaram valores superiores a $500 \mathrm{NMP} / \mathrm{g}$, acima do limite permitido pela legislação(BRASIL, 2001a), sendo 76,47\% (26/34) dos queijos de coalho com inspeção estadual, $100 \%$ (1/1) com inspeção federal e 100\% (7/7) sem inspeção (Tabela 2). Evidenciou-se a presença de E. coli em $64,29 \%$ (27/42) das amostras, sendo $64,71 \%$ (22/34) nos queijos com inspeção estadual e 71,43\% (5/7) nos sem inspeção. Segundo LeIte et al. (2002), emSalvador, BA, 90,62\% (29/32) das amostras de queijo de coalho analisadas encontravam-se em condições impróprias para consumo, por apresentarem valores acima dos limites estabelecidos pela legislação vigente para coliformes termotolerantes, dos quais 37,50\% (12/32) foram identificadas como E. coli. Estudos realizados no Estado do Ceará, por BORGES et al. (2003), confirmaram a presença de coliformes totais e coliformes fecais em todas as amostras de queijos avaliadas, sendo que $74,4 \%$ das amostras continham níveis superiores aos padrões estabelecidos pela legislação vigente para coliformes termotolerantes, com confirmação de E. coli em 93\% das amostras. DuARTE et al. (2005), analisando a presença de micro-organismos indicadores das condições higiênico-sanitárias em queijo de coalho no Estado de Pernambuco, detectaram coliformes termotolerantes em valores acima do aceitável em 44,10\% das amostras, enquanto FerTosA et al. (2003) identificaram, em $36,4 \%$ das amostras de queijo de coalho, coliformes fecais entre 3 e $7 \mathrm{NMP} / \mathrm{g}$, com confirmação de $E$. coli, porém, dentro dos limites estabelecidos pela legislação.

Tabela 1 - Frequência de aceitabilidade microbiológica conforme RDC 12 por procedência de Inspeção Sanitária em queijos de coalho comercializados no Município do Cabo de Santo Agostinho, PE, durante o período de janeiro a dezembro de 2008.

\begin{tabular}{|c|c|c|c|c|c|c|c|c|}
\hline \multirow{3}{*}{$\begin{array}{l}\text { Aceitabilidade microbiológica } \\
\text { (RDC 12) }\end{array}$} & \multicolumn{8}{|c|}{ Amostra } \\
\hline & \multicolumn{2}{|c|}{$\begin{array}{c}\text { Inspeção federal } \\
\text { (tipo A) }\end{array}$} & \multicolumn{2}{|c|}{$\begin{array}{c}\text { Inspeção estadual } \\
\text { (tipo B) } \\
\end{array}$} & \multicolumn{2}{|c|}{$\begin{array}{c}\text { Sem inspeção } \\
\text { (sem rótulo) }\end{array}$} & \multicolumn{2}{|c|}{ Total } \\
\hline & $\mathrm{N}^{\circ}$ & $\%$ & $\mathrm{~N}^{\circ}$ & $\%$ & $\mathrm{~N}^{\circ}$ & $\%$ & $\mathrm{~N}^{\circ}$ & $\%$ \\
\hline Inaceitável & 0 & 100 & 32 & 94,12 & 7 & 100 & 40 & 95,24 \\
\hline Aceitável & 0 & 0,00 & 2 & 5,88 & 0 & 0,00 & 2 & 4,76 \\
\hline Total & 1 & 100 & 34 & 100 & 7 & 100 & 42 & 100 \\
\hline
\end{tabular}

Tabela 2 - Frequência do número mais provável (NMP/g) de coliformes termotolerantes por procedência de inspeção sanitária em queijos de coalho comercializados no Município do Cabo de Santo Agostinho, PE, durante o período de janeiro a dezembro de 2008.

\begin{tabular}{lcccccccc}
\hline \multirow{2}{*}{$\begin{array}{c}\text { NMP/g de Coliformes Ter- } \\
\text { motolerantes }\end{array}$} & $\begin{array}{c}\text { Anspeção federal } \\
\text { (tipo A) }\end{array}$ & $\begin{array}{c}\text { Inspeção estadual } \\
\text { (tipo B) }\end{array}$ & $\begin{array}{c}\text { Sem inspeção } \\
\text { (sem rótulo) }\end{array}$ & Total \\
\cline { 2 - 9 } & $\mathrm{N}^{\circ}$ & $\%$ & $\mathrm{~N}^{\circ}$ & $\%$ & $\mathrm{~N}^{\circ}$ & $\%$ & $\mathrm{~N}^{\circ}$ & $\%$ \\
\hline 23 a 460 & 0 & 0,00 & 8 & 23,53 & 0 & 0,00 & 8 & 19,05 \\
$\geq 1100$ & 1 & 100 & 26 & 76,47 & 7 & 100 & 34 & 80,95 \\
\hline Total & 1 & 100 & 34 & 100 & 7 & 100 & 42 & 100 \\
\hline
\end{tabular}

RDC $12 \leq 500 \mathrm{NMP} / \mathrm{g}$. 
É importante destacar que coliformes termotolerantes pertencem a um grupo de micro-organismos que habita o trato intestinal do homem e de outros animais, portanto sua presença em alimentos indica que houve contato direto ou indireto do produto com fezes, evidenciando risco para a saúde dos consumidores devido sua alta patogenicidade (SALOTTI et al., 2006).

No que se refere ao Staphylococcus coagulase positiva, 76,19\% (32/42) das amostras apresentaram contagens variando entre $1,6 \times 10^{3}$ a 2,0 $010^{5} \mathrm{UFC} / \mathrm{g}$ (Tabela 3.), contrariando a RDC 12 que tolera, no máximo, 5,0 × $10^{2} \mathrm{UFC} / \mathrm{g}$ para esta bactéria (BRASIL, 2001a). Foram classificados como impróprios para o consumo humano $79,41 \%$ (27/34) dos queijos de coalho com inspeção estadual (tipo B) e 71,43\% (5/7) dos sem inspeção. Notou-se que a amostra de queijo com inspeção federal, produzido com leite pasteurizado (tipo A), apresentou contaminação, por este micro-organismo, em nível tolerável (Tabela 3). A ocorrência de Staphylococcus coagulase positiva foi observada em $93,1 \%$ das amostras de queijo de coalho analisadas no Estado do Ceará, sendo que $90,8 \%$ destas enquadraram-se fora dos padrões microbiológicos vigentes para este patógeno e foram classificados como produto em condições higiênico-sanitárias insatisfatórias, segundo relatos de Borges et al. (2003). Estudos realizados para identificar os aspectos microbiológicos do queijo de coalho comercializado em Fortaleza, CE, identificaram discordância sanitária para Staphylococcus coagulase positiva em $62,5 \%$ das amostra (SANTOS et al., 1995). No Estado do Rio Grande do Norte foi observado $S$. aureus em $72,7 \%$ das amostras de queijo de coalho, com contagens variando de 7,0 x $10^{4}$ a $1,3 \times 10^{8} \mathrm{UFC} / \mathrm{g}$ (Feitosa et al., 2003). Em discrepância com os nossos resultados, SALOTTI et al. (2006) identificaram Staphylococcus coagulase positiva em apenas $15 \%$ das amostras inspecionadas e $20 \%$ das sem inspeção nos queijos Minas Frescal comercializados no Município de Jaboticabal, SP, contagens superiores ao permitido pela legislação vigente.

Segundo Carmo; Bergdoll (1990), alimentos com contagens de Staphylococcus coagulase positiva variando entre $10^{4}$ a $10^{8} \mathrm{UFC} /$ gjá apresentaram enterotoxinas. No caso específico do leite, os tratamentos térmicos disponíveis não são capazes de inativar estas enterotoxinas, constituindo risco potencial para o consumidor (LAMAITA et al., 2005).

Tabela 3 - Frequência de Unidade Formadora de Colônia (UFC/g) de Staphylococcus coagulase positiva por procedência de inspeção sanitária em queijos de coalho comercializados no Município do Cabo de Santo Agostinho, PE, entre janeiro e dezembro de 2008.

\begin{tabular}{|c|c|c|c|c|c|c|c|c|}
\hline \multirow{3}{*}{$\begin{array}{c}\text { UFC/g de Staphylococcus coagulase } \\
\text { positiva }\end{array}$} & \multicolumn{8}{|c|}{ Amostra } \\
\hline & \multicolumn{2}{|c|}{$\begin{array}{l}\text { Inspeção federal } \\
\text { (tipo A) }\end{array}$} & \multicolumn{2}{|c|}{$\begin{array}{l}\text { Inspeção estadual } \\
\text { (tipo B) }\end{array}$} & \multicolumn{2}{|c|}{$\begin{array}{c}\text { Sem inspeção } \\
\text { (sem rótulo) }\end{array}$} & \multicolumn{2}{|c|}{ Total } \\
\hline & $\mathrm{N}^{\circ}$ & $\%$ & $\mathrm{~N}^{\circ}$ & $\%$ & $\mathrm{~N}^{\circ}$ & $\%$ & $\mathrm{~N}^{\circ}$ & $\%$ \\
\hline$<10$ & 1 & 100 & 7 & 20,59 & 2 & 28,57 & 10 & 23,81 \\
\hline $1,6 \times 10^{3}$ a $8 \times 10^{3}$ & 0 & 0,00 & 2 & 5,88 & 1 & 14,29 & 3 & 7,14 \\
\hline $1,2 \times 10^{4}$ a $2 \times 10^{4}$ & 0 & 0,00 & 20 & 58,82 & 3 & 42,85 & 23 & 54,76 \\
\hline $1,2 \times 10^{5}$ a $2 \times 10^{5}$ & 0 & 0,00 & 5 & 14,71 & 1 & 14,29 & 6 & 14,29 \\
\hline Total & 1 & 100 & 34 & 100 & 7 & 100 & 42 & 100 \\
\hline
\end{tabular}

RDC $12 \leq 5 \times 10^{2} \mathrm{UFC} / \mathrm{g}$.

Tabela 4 - Frequência de Salmonella por procedência de inspeção sanitária em queijos de coalho comercializados no Município do Cabo de Santo Agostinho, PE, entre janeiro e dezembro de 2008.

\begin{tabular}{|c|c|c|c|c|c|c|c|c|}
\hline \multirow{3}{*}{ Salmonella sp./25g } & \multicolumn{8}{|c|}{ Amostra } \\
\hline & \multicolumn{2}{|c|}{$\begin{array}{c}\text { Inspeção federal } \\
\text { (tipo A) }\end{array}$} & \multicolumn{2}{|c|}{$\begin{array}{c}\text { Inspeção estadual } \\
\text { (tipo B) }\end{array}$} & \multicolumn{2}{|c|}{$\begin{array}{c}\text { Sem inspeção } \\
\text { (sem rótulo) }\end{array}$} & \multicolumn{2}{|c|}{ Total } \\
\hline & $\mathrm{N}^{\circ}$ & $\%$ & $\mathrm{~N}^{\circ}$ & $\%$ & $\mathrm{~N}^{\circ}$ & $\%$ & $\mathrm{~N}^{\circ}$ & $\%$ \\
\hline Ausência & 1 & 100 & 30 & 88,24 & 7 & 100 & 38 & 90,48 \\
\hline Presença & 0 & 0,00 & 4 & 11,76 & 0 & 0.00 & 4 & 9,52 \\
\hline Total & 1 & 100 & 34 & 100 & 7 & 100 & 42 & 100 \\
\hline
\end{tabular}

RDC 12 = Ausência Salmonella sp./25g. 
MARQues et al. (2006) isolaram 70 cepas de Staphylococcus coagulase positiva em queijo minas frescal e leite de vacas com mastite através de provas bioquímicas, sendo o $S$. aureus a que predominou com 36 cepas. RAPINI et al. (2004) verificaram elevada frequência de resistência das cepas de Staphylococcus sp. isoladas de amostras de queijo de coalho comercializadas em praias nordestinas frente aos antibióticos utilizados em medicina humana e veterinária.

S. aureus é um patógeno responsável por intoxicações que resultam da ingestão de alimentos contaminados por enterotoxinas termoestáveis e pré-formadas (Su; WoNG, 1997).

Quanto à Salmonella sp., 9,52\% (4/42) dos queijos analisados apresentaram presença deste microorganismo (Tabela 4). Valores semelhantes foram identificados no Estado do RioGrande do Norte, onde em $9 \%$ das amostras foram positivas para o isolamento de Salmonella sp. (Feitosa et al., 2003). Duarte et al. (2005) detectaram presença desta bactéria em $5,5 \%$ das amostras de queijos coletadas em estabelecimentos comerciais no Estado de Pernambuco. Percentual superior foi encontrado por BorGEs et al. (2003), que constataram a presença deste micro-organismo em $34,9 \%$ da amostra de queijos de coalho produzidos no Estado do Ceará. A legislação brasileira (BRASIL, 2001a) não permite a presença desta bactéria em alimentos.

NADVORnY et al. (2004), avaliando a ocorrência de DTA no Rio Grande do Sul, constataram que 74,7\% dos surtos ocorridos no ano 2000 foram ocasionados por Salmonella sp. eque a utilização de matéria-prima sem inspeção sanitária e a manipulação incorreta dos alimentos constituíram-senos fatores predisponentes à contaminação dos alimentos.

Segundo Ávila; Gallo (1996), os produtos de laticínios são um dos mais importantes veículos de transmissão de Salmonella sp. e o consumo de queijo de coalho contaminado por esta bactéria pode representar risco à saúde da população nordestina, visto que este é um alimento típico e acessível à maioria das classes sociais do Nordeste (DuARTE et al., 2005).

\section{CONCLUSÃO}

A maioria dos queijos de coalho comercializados no Município do Cabo de Santo Agostinho, Estado de Pernambuco, Brasil, apresenta-se em desacordo com os padrões microbiológicos vigentes na legislação brasileira, sendo considerada imprópria para o consumo humano.

\section{AGRADECIMENTOS}

Ao Laboratório Central de Saúde Pública de Pernambuco (LACEN), por disponibilizar todo aparato laboratorial para pesquisa, principalmenteà supervisora de bromatologia e toxicologia Dra. Lúcia Roberta e ao analista em saúde Dr. Sandoval Filho, pela grande contribuição prestada nas análises laboratoriais; À Prefeitura do Cabo de Santo Agostinho e em especial à equipe da Vigilância Sanitária, por toda ajuda durante a execução do trabalho.

\section{REFERÊNCIAS}

\section{AMERICAN PUBLIC HEALTH ASSOCIATION.}

Compendium of methods for the microbiological examination of foods. DOWNES, F.P.; ITO, K. (Ed.). 4. ed.

Washington: APHA, 2001.676p.

ÁVILA, C.R.; GALLO, C.R. Pesquisa de Salmonella spp. em leite cru, leite pasteurizado tipo $C$ e "queijo minas frescal" comercializado no Município de Piracicaba SP. Scientia Agricola, v.53, n.1, p.159-163, 1996.

BORGES, M.F.; FEITOSA, T.; NASSU, R.T.; MUNIZ, C.R.; AZEVEDO, E.H.F.; FIGUEIREDO, E.A.T. Microrganismos patogênicos e indicadores em queijo coalho produzido no Estado do Ceará, Brasil. Boletim do Centro de Pesquisa e Processamento de Alimentos, v.21, n.1, p.3140, 2003.

BRASIL. Ministério da Saúde. Resolução da Diretoria Colegiada no 12 de 02 de janeiro de 2001. Regulamento Técnico sobre Padrões Microbiológicos para Alimentos. Diário Oficial [da] República Federativa do Brasil, Brasília, 02 de janeiro de 2001, seção I, p. 45-53, 2001a.

BRASIL. Ministério da Agricultura, Pecuária e Abastecimento. Instrução normativa $n^{\circ} 30$ de 26 de junho de 2001. Regulamentos Técnicos de Identidade e Qualidade de Manteiga da Terra ou Manteiga de Garrafa; Queijo de Coalho e Queijo de Manteiga. Diário Oficial [da] República Federativa do Brasil, Brasília, 16 de julho de 2001, seção I, p. 13, 2001 b.

CARMO, L.S.; BERGDOLL, M.S. Staphylococcal food poisoning in Belo Horizonte (Brasil). Revista de Microbiologia, v.21, n.4, p.320-323, 1990.

CAVALCANTE, J.F.M.; ANDRADE, N.J.; FURTADO, M.M.; FERREIRA, C.L.L.F.; PINTO, C.L.O.; ELARD, E. Processamento de queijo coalho regional empregando leite pasteurizado e cultura lática endógena. Ciência e Tecnologia de Alimentos, v.27, n.1, p.205-214, 2007.

CORREIA, M.; RONCADA, M.J. Caracterização microscópica de queijo de prato, mussarela e mineiro comercializados em feiras livres da cidade de São Paulo. Revista de Saúde Pública, v.31, n.3, p. 296-301, 1997.

CPRM - SERVIÇO GEOLÓGICO DO BRASIL. Projeto cadastro de fontes de abastecimento por água subterrânea, Pernambuco: Diagnóstico do Município de Cabo de Santo Agostinho. Recife: CPRM, 2005. 11p. 
DUARTE, D.A.M.; SCHUCH, D.M.T.; SANTOS, S.B.; RIBEIRO, A.R.; VASCONCELOS, A.M.M.; SILVA, J.V.D.; MOTA, R.A. Pesquisa de Listeria monocytogenes e microrganismos indicadores higiênicos-sanititários em queijo de coalho produzido e comercializado no Estado de Pernambuco. Arquivos do Instituto Biológico, São Paulo, v.72, n.3, p.297-302, 2005.

FEITOSA, T.; BORGES, M.F.; NASSU, R.T.; AZEVEDO, E.H.F.; MUNIZ, C. R. Pesquisa de Salmonella sp., Listeria sp. e microrganismos indicadores higiênicos-sanitários em queijos produzidos no Estado do Rio Grande do Norte. Ciência e Tecnologia de Alimentos, v.23, p.162-165, 2003. Suplemento 1.

INSTITUTO BRASILEIRO DE GEOGRAFIA E ESTATÍSTICA. Contagem da população 2007 e estimativa da população 2007. Brasília, [2007]. Disponível em: <http:/ / www.ibge.gov.br/home/estatistica/populacao/contagem2007/PE.pdf > Acesso em: 15 set. 2008.

LAMAITA, H.C.; CERQUEIRA, M.M.O.P.; CARMO, L.S.; SANTOS, D.A.; PENNA, C.F.A.M.; SOUZA, M.R. Contagem de Staphylococcus sp. e detecção de enterotoxinas estafilocócicas e toxina da síndrome do choque tóxico em amostra de leite cru refrigerado. Arquivo Brasileiro de Medicina Veterinária e Zootecnia, v.57, n.5, p.702-709, 2005.

LEITE, C.C.; GUIMARÃES, A.G.; RIBEIRO, N.S.; SILVA, M.D.; ASSIS, P.N. Pesquisa de Listeria monocytogenes e Escherichia coli em queijo do tipo "coalho" comercializado em Salvador (BA). Importância para saúde pública. Revista Analytica, n.2, p.8-41, 2002.

LIMA, M.H.P.; TELLES, F.J.S.; MACEDO, B.A.; BENEVIDES, S.D. Elaboração de queijo de Coalho a partir de leite pasteurizado e inoculado com Streptococcus thermophyllus e Lactobacillus bulgaricus: Aspectos bioquímico e sensorial. Boletim do Centro de Pesquisa e Processamento de Alimentos, v.16, n.1, p.37-44, 1998.

MANGUEIRA, T.F.B.; TRAVASSOS, A.E.R.; MOREIRA, R.T. Teste de aceitabilidade sensorial de queijo de coalho com baixo teor de gordura enriquecido com ferro. Boletim do Centro de Pesquisa e Processamento de Alimentos, v.20, n.2, p.279-290, 2002.

MARQUES, M.R.H.; MARTINS, R.P.; CUNHA NETO, A. Ocorrência de Staphylococcus coagulase positiva em leite e queijo: identificação, perfil enzimático e biotipagem. Higiene Alimentar, v.20, n.140, p.86-94, 2006.

NADVORNY, A.; FIGUEIREDO, D.M.S.; SCHMIDT, V. Ocorrência de Salmonella sp. em surtos de doenças transmitidas por alimentos no Rio Grande do Sul em 2000. Acta Scientiae Veterinariae, v.32, n.1, p.47-51, 2004.

PERESI, J.T.M.; ALMEIDA, I.A.Z.C.; LIMA, S.I.; MARQUES, D.F.; RODRIGUES, E.C.A.; FERNANDES, S.A.; GELLI, D.S.; IRINO, K. Surtos de enfermidades transmitidas por alimentos causados por Salmonella Enteritidis. Revista de Saúde Pública, v.32, n.5, p.477-483, 1998.

PERNAMBUCO. Secretaria Produção de Rural e Reforma Agrária. Resolução n. 002 de 19 de abril de 1999. Estabelece a identidade e os requisitos mínimos de qualidade que deverá cumprir o Queijo Coalho produzido no Estado de Pernambuco e destinado ao consumo humano. Diário Oficial do Estado de Pernambuco, Recife, 20 de abril de 1999.

RAPINI, L.S.; TEIXEIRA, J.P.; MARTINS, N.E.; CERQUEIRA, M.M.O.P.; SOUZA, M.R.; PENNA, C.F.A.M Perfil de resistência antimicrobiana de cepas de Staphylococcus sp. isoladas de queijo tipo coalho. Arquivo Brasileiro de Medicina Veterinária e Zootecnia v.56, n.1, p.130-133, 2004.

SALOTTI, B.M.; CARVALHO, A.C.F.B.; AMARAL, L.A.; VIDAL-MARTINS, A.M.C.; CORTEZ, A.L. Qualidade microbiológica do queijo minas frescal comercializado no Município de Jaboticabal, SP, Brasil. Arquivos do Instituto Biológico, São Paulo, v.73, n.2, p.171-175, 2006.

SALVADOR, M.; CAMASSOLA, M.; MOSCHEN, ESTER, S; ZANROSSO, A.V. Avaliação da qualidade microbiológica do queijo de prato e parmesão ralado. Boletim do Centro de Pesquisa e Processamento de Alimentos, v.19, n.1, p.65-74, 2001.

SANTOS, F.A.; NOGUEIRA, N.A.P; CUNHA, G.M.A. Aspectos microbiológicos do queijo tipo coalho comercializado em Fortaleza - Ceará. Boletim do Centro de Pesquisa e Processamento de Alimentos, v.13, n.1, p.31-361, 1995.

SERVIÇO BRASILEIRO DE APOIO ÀS MICRO E PEQUENAS EMPRESAS. Queijo coalho: aspectos técnicos de produção. Recife: SEBRAE-PE, 1994. 44p. (Série Agroindústria)

SU, Y.C.; WONG, A.C.L. Current perspectives on detection of Staphylococcal enterotoxins. Journal of Food Protection, v.60, n.2, p.195-202, 1997.

Recebido em 20/5/09

Aceito em 26/7/10 